\title{
Public health inequalities, structural missingness, and digital revolution: time to question assumptions
}

\author{
Elena N. Naumova ${ }^{1}$
}

Accepted: 28 October 2021 / Published online: 22 November 2021

(c) The Author(s), under exclusive licence to Springer Nature Limited 2021

Over my career in public health as a data scientist, I increasingly encountered a persistent problem: populations that need the most attention of public health professionals are the populations about which we have the least information. People need attention because of their ongoing health concerns or risks and hazards imposed by society and the environment. The information, data, and thus the knowledge about these groups are often incomplete, inconsistent, outdated, and simply do not exist. And the data 'missingness' is something we systematically avoid discussing in publications, data analyses, algorithm development, policymaking. Our common assumption about missing data is that their effect on our scientific findings and practical decisions is inconsequential. We typically assume that research designs, statistical tools, logical analysis, and technological advances are immune to data flaws. We often assume that the missing data are rare and random. Or, if not, that we are well equipped to handle this problem.

I question these assumptions. I am convinced that with the growing health inequalities and the booming digital revolution we should pay attention to the challenges posed by missing data in public health research and practice.

Missing data have many forms. 'Missing' might mean unknown, undetected, undetermined, not applicable, lost, replaced by a blank due to an error. Data could be missing because of malfunctioning equipment, or because of mistakes in data handling (documenting, recording, reporting, correcting), or because the population seemed to be inaccessible. Worldwide the growing public health databases collect information on broadly accepted determinants of health-from demographic characteristics to socioeconomic status. Many conclusions in public health research stem from self-reported surveys and questionaries that ask participants of nationally representative cohorts about their income, education, sexual orientation. Yet complexities in presenting the information related to gender, race, ethnicity, country of origin or resettlement, education, occupation, past medical and vaccination histories are staggering. We are challenged with questions on how to reflect gender fluidity,

Elena N. Naumova

elena.naumova@tufts.edu

1 Friedman School of Nutrition Science and Policy, Tufts University, Medford, USA 
to code multiple races and ethnicity, to ensure medical terms and questionaries are properly translated in various languages, to ensure the questions are understood and not ignored, to ensure records are timely delivered to a data repository. It is no secret that in many instances, the easiest solution to anything uncommon, unusual, unconforming is to leave such information blank. Responders tend to leave the answers blank when questions are poorly structured, confusing, or carry a stigma. Researchers tend to leave data blank when the population is hard-to-reach.

The disproportional missingness and poor reporting of health-related data in the hard-to-reach populations have not happened at random. It is structural missingness. As society imposes and reinforces structural vulnerability, the instruments we use to gather and process data impose structural missingness. In hard-to-reach populations, these questions stemmed from economic inequalities that also remind the participants why they are disfranchised, marginalized, stigmatized. Emotional distress prompted by these questions may dictate reactions among the responders. Imagine how someone sidelined would respond when bombarded with questions about their poverty, incomplete education, and need for welfare. Why should public health professionals or anyone else asking such questions already associated with stigma expect high response rates or compete reliable answers? We don't. Together with data scientists we create data imputation algorithms to fill the holes, we drop incomplete data from analyses, we render those who are hard to reach invisible.

Worldwide, the pandemic has amplified existing structural vulnerabilities and made the path to reach those who are vulnerable much harder. While expanding the context for understanding and addressing diversity and inequalities, we recognize that the fraction of people with depleted resources - migrants, refugees, those resettled-is continuing to grow and to expand the pool of the hard-to-reach. No question that the efforts to implement large-scale national surveys and outbreak tracking systems across the world are commendable. Yet, the foundational inequalities existed prior to the pandemic. The ongoing data collection efforts inherit all flaws and, often, inabilities to capture hard-to-reach populations.

The digital revolution offers a promise to address many issues in public health by designing global interoperable surveillance systems, comprehensive data repositories of electronic medical records, smart sensors, and wearable devices. The digital transformation should help to build agreements among data platform developers, supporting institutions and agencies, decision-makers, and public health policy experts on what, how, where, and when data should be collected, preserved, assessed, and shared. The argument that the world is ready for a digital transformation is that the health data provided by the official statistics at the national and community levels are capturing populations with high fidelity, granularity, and representativeness. This view needs to be carefully reexamined.

The digital revolution in healthcare is already quietly taking over the world. It is reallocating resources, dictating the need for a workforce, captivating bystanders with 'shiny apps', and promising a bright future. Some argue that the digital transformation of health systems will solve health inequity problems. The moguls of information technology promise to address inequity in accessing reliable health information, medical expertise, and equipment for individuals otherwise disenfranchised from care. The developers promise to improve representation by shifting 
from paper-based documentation to Artificial Intelligence (AI)-powered dashboards, increasing access to health services by enabling telemedicine, and by implementing smart innovative solutions. Investors promise that health information systems and the data they collect will equitably represent those who are marginalized, who live in vulnerable communities and conflict-torn regions, those who are hard to reach.

I want to believe in these promises. In fact, I am part of this data analytics world by training. I am one of those who analyze data, build models, and create algorithms for public health applications. Teaching in the graduate programs for public health professionals, I emphasize five essential types of the knowledge gap that powered innovations. The first type (1) addresses the existential gap when the data, information, and models that are needed to tackle a specific problem do not exist-when the problem is so new that many elements require a fresh look. To some extent, this type of gap accompanies any rare event - the ongoing pandemic is a good examplewhen we must learn as we go through the process. The remaining four types are more common. They occur when data, information, and models are (2) incomplete, (3) inconclusive, (4) inconsistent, or (5) outdated. In other words when data are suffering from various forms of 'missingness'. The ability to recognize and address these gaps helps researchers and practitioners to recognize pressing questions, formulate sound hypotheses, and power intelligent solutions. These gaps also should drive the digital transformation in public health by adopting new technologies and supporting policies with more reliable, complete, and systematically updated information and evidence-based solutions.

Data missingness is the silent feature that biases AI algorithms. Human intelligence, knowledge, and the will to solve health problems are behind AI applied to public health needs. Digital technologies depend heavily on rules designed by humans. These rules dictate the behaviors and capabilities of digital technologies. If we know little or nothing about the public health issues in the hard-to-reach populations, these issues do not exist-at least in AI applications-because no data support the rules implemented in those AI applications. In building such applications we often assume that what is missing is negligible, happens rarely, by chance, randomly. When the reasons for missingness vary in well-off and marginalized groups, it would be naïve to assume that the consequences of missingness are identical across diverse populations. So far, is it not apparent how well the digital revolution serves public health needs, how well it addresses health inequalities, or knowledge gaps related to inequalities. It is much clearer so far in how it serves the financial management of medical services.

While societal inequalities are growing, the information gap in knowledge that could power the solutions both locally and globally is growing as well. What we know about inequality becomes outdated fast. What we want to know in greater detail is often obscured by sweeping aggregations and generalizations. At the individual level, those details are often seen as stigmatizing; multiplied by the survey-taking fatigue they prompt low response rates. At the national level, data related to health status, disease prevalence rates, participation in global surveillance efforts, when shared with global audiences and international organizations, reflect glaring inequalities across countries [1]. There is no question that transformational changes are essential to optimize resource allocation, improve efficiency 
and effectiveness of public health interventions, and identify and implement strategies to achieve sustainability. Yet, to be truly effective and sustainable, the preferred focus of digital technologies should be shifted to disease prevention, aiming to promote and support a healthy lifestyle and thus, to reduce the existing and future burdens on healthcare.

The current view of the digital industry promising to improve the assess to trustworthy health information is that at an individual level, misinformation and disinformation form the barriers to a person's ability to pursue health. At best, this view is naïve. Digital tools could play an enormous role in satisfying the need for reliable health information. Yet the information crisis of the ongoing pandemic continues to fuel the 'infodemic'. The digital world is failing to promote trusted science, clarify the value of vaccination, and protect health workers from bullying and harassment. Such failure highlights the glaring gaps in ethics surrounding technical solutions.

Structural data missingness and gaps in ethical considerations mimic the socio-economic gaps and structural disparities. It is not yet clear how the digital revolution and AI could help to bridge the divide. One of the solutions is to make AI health systems transparent, so the predictive models could explain themselves in ways that humans can understand, as proposed by Cynthia Rudin, the recent recipient of the Squirrel AI Award for Artificial Intelligence for the Benefit of Humanity [2]. By making health information clear and transparent, we could incorporate accountability and corrective actions.

What is the role of public health professionals in solving health inequalities as part of a digital revolution promise? To ensure proper allocation of resources to programs to reach out to the marginalized populations, seek solutions, strategies, and best practices to minimize data missingness, we must ensure the voices of those who are hard-to-reach are heard and their issues are recognized by policymakers and the society at large. At a minimum, we can improve standards for data reporting, enforce data completeness and quality, ensure transparency in handling data missingness $[3,4]$.

The pledge for improving public health implies efforts to reduce health inequality. As health inequalities continue to grow, this pressing topic captures the attention of many in social media, advocacy groups, and academic fields along with their counterparts in business sectors. "Attention is the beginning to devotion," said Mary Oliver, the Pulitzer Prize-winning poet. Devotion and commitment to public health is a must to ensure that the ongoing digital revolution serves everyone by making population-level health responses quicker, more inclusive, more effective, more robust, and less prone to errors.

Elena N. Naumova, Editor-in-Chief 


\section{References}

1. Simpson RB, Gottleib J, Zhou B, Hartwick MA, Naumova EN. Completeness of open access FluNet surveillance data. Sci Rep. 2021;11:795.

2. AAAI's Squirrel AI Award for Artificial Intelligence to Benefit Humanity. 2021. https://aaai.org/ Pressroom/Releases/release-21-1012.php. Accessed 24 Oct 2021.

3. Krobath DM, Taylor SF. A review of engaging black and minority ethnic groups in health research: 'Hard to Reach'? Demystifying the misconceptions. J Public Health Policy. 2021;42(4).

4. Darko N. Engaging black and minority ethnic groups in health research: 'Hard to Reach'? Demystifying the misconceptions. Policy Press, United Kingdom. (ISBN: 1447359127) 2021.

Publisher's Note Springer Nature remains neutral with regard to jurisdictional claims in published maps and institutional affiliations. 